\title{
Analysis of disease progression and prognosis in differentiated thyroid cancer with pulmonary metastases: a retrospective study
}

\author{
Hiroyuki Iwasaki, MD*, Soji Toda, MD, Daisuke Murayama, MD, Ai Matsui, MD
}

\begin{abstract}
Introduction: Pulmonary metastasis originating from differentiated thyroid cancer is rare. Pulmonary metastasis generally progresses slowly and results in a relatively long prognosis when treated with radioactive iodine therapy and thyroid-stimulating hormone suppression therapy. However, some cases still result in death. Since 2015, lenvatinib administration for pulmonary metastases with disease progression has yielded satisfactory results.

Materials and methods: Among the 798 patients with differentiated thyroid cancer treated at Kanagawa Cancer Center, Japan, between April 2015 and March 2020, 194 had distant metastasis. Of these 194 patients, 118 diagnosed with pulmonary metastasis had lesions that influence the prognosis. We retrospectively investigated the transition of the maximum diameter of pulmonary metastases, serum thyroglobulin, follow-up, and survival time.

Results: We included 83 follow-up cases and 35 patients treated with lenvatinib. Considering that the disease progressed, 35 patients were treated with lenvatinib, and 4 died from cancer-specific disease. Treatment results were evaluated as progressive disease, stable disease, and partial response in 2, 11, and 22 patients, respectively.

Conclusions: Among pulmonary metastases, no death occurred because of relatively slow disease progression up to a maximum diameter of $10 \mathrm{~mm}$. However, when the size exceeded $15 \mathrm{~mm}$, radioactive iodine treatment and thyroid-stimulating hormone suppression therapy did not work, and disease progression accelerated. As long as the lenvatinib treatment could be continued, the disease could be controlled satisfactorily. The patients who discontinued lenvatinib died from disease progression.
\end{abstract}

Keywords: Differentiated thyroid cancer, Pulmonary metastasis, Lenvatinib, Prognosis

The prognosis for differentiated thyroid cancer (DTC) is relatively good, and a papillary microcarcinoma smaller than $1 \mathrm{~cm}$ seldom requires surgical treatment ${ }^{[1,2]}$. The prevalence rate of distant metastases from DTC is $2.2 \%-3.1 \%{ }^{[3,4]}$. We treated 798 patients with DTC for 5 years from April 2015 to March 2019 at Kanagawa Cancer Center, Japan, and 194 of them (24.3\%) had distant metastases. Considering institutional bias, many referrals for advanced thyroid cancer and/or distant metastases from DTC were recorded. In fact, 42 of 194 DTC patients with distant metastases died during this 5 -year period. Among distant metastases, pulmonary metastases are the most common $^{[5]}$.

Department of Breast and Endocrine Surgery, Kanagawa Cancer Center, Yokohama City, Japan

This manuscript has been peer reviewed.

Sponsorships or competing interests that may be relevant to content are disclosed at the end of this article.

*Corresponding author. Address: Department of Breast and Endocrine Surgery, Kanagawa Cancer Center, 2-3-2 Nakao Asahi-Ku, Yokohama City 241-8515, Japan. Tel: + 81-45-520-2222; fax: +81-45-520-2202. E-mail address: iwasaki. h@kcch.jp (H. Iwasaki).

Copyright (C) 2020 The Authors. Published by Wolters Kluwer Health, Inc. on behalf of IJS Publishing Group Ltd. This is an open-access article distributed under the terms of the Creative Commons Attribution-Non Commercial License 4.0 (CCBYNC), where it is permissible to download, share, remix, transform, and buildup the work provided it is properly cited. The work cannot be used commercially without permission from the journal.

International Journal of Surgery Oncology (2020) 5:e104

Received 7 September 2020; Accepted 7 October 2020

Published online 9 November 2020

http://dx.doi.org/10.1097//J9.0000000000000104
Disease progression can be accurately diagnosed using the computed tomographic (CT) images of pulmonary metastases. Before tyrosine kinase inhibitor (TKI) treatment for distant metastases, the 5 -year survival rate was $\sim 50 \%^{[6]}$. We hypothesized that lenvatinib can prevent the progression of pulmonary metastases, thereby prolonging death from disease progression or anaplastic transformation. In this study, we aimed to thoroughly examine the outcomes of patients with pulmonary metastases treated with lenvatinib.

\section{Materials and methods}

The Chemotherapy Committee of Kanagawa Cancer Center approved the lenvatinib treatment regimens administered to patients with DTC in this study. The Cancer Board of the hospital also approved these TKI treatments, including surgery, for patients with DTC who were included. The study was approved by the Institutional Review Board of Kanagawa Cancer Center (IRB approval number: 27-61). This study is retrospective in design. Among the 194 DTC patients with distant metastases, 155 had pulmonary metastases, 46 had bone metastases, 61 had lymph node recurrence, and 45 had local recurrence. Furthermore, 118 of these 194 patients were included in this study in which we assessed that their pulmonary metastasis influenced their prognosis. We found that 71 patients with DTC had only pulmonary metastases. When lesions were found in multiple organs, the target lesion was decided based on the RECIST guideline ${ }^{[7]}$ when the tumor burden of pulmonary metastases was large. There were 47 cases with more tumor burden of other lesions; these cases were excluded from the study. 
Of the 118 cases, 83 were under observation, 51 with maximum diameter of metastases of $<10 \mathrm{~mm}$ were in the small metastasis (SM) group, 32 with maximum diameter of metastases of $\geq 10$ $\mathrm{mm}$ were in the large metastasis (LM) group, and disease progression was noted. Further, 35 cases using lenvatinib were designated as the lenvatinib treatment (LV) group. Table 1 summarizes patient characteristics. The treatment strategy for distant metastases included total thyroidectomy, maximum of 6 times of radioactive iodine (RAI) therapy as far as effective, concurrent thyroid-stimulating hormone (TSH) suppression therapy, and examination of the presence or absence of disease progression using the serum thyroglobulin level and antibodies and CT image. A total of 35 patients who received RAI treatment but became refractory with disease progression consented to receive lenvatinib treatment. All patients provided informed consent for using their samples for medical investigation and clinical research. This study was approved by the Institutional Review Board of Kanagawa Cancer Center (IRB approval number: 27-61).

\section{Statistical analysis}

The median values between 2 groups were compared using the Fisher test for nominal variables and the Mann-Whitney $U$ test for continuous variables. The statistically significant difference was set at $P<0.05$. All statistical data were analyzed using the $\mathrm{EZR}^{[8]}$. The overall survival (OS) was calculated using the

\section{Table 1}

Patient characteristics of the follow-up and lenvatinib treatment groups.

\begin{tabular}{|c|c|c|c|c|}
\hline Group & $\begin{array}{c}\text { Small } \\
\text { Metastasis }\end{array}$ & $\begin{array}{c}\text { Large } \\
\text { Metastasis }\end{array}$ & Lenvatinib & $P$ \\
\hline $\mathrm{N}$ & 51 & 32 & 35 & \\
\hline T0 (\%) & $1(2.0)$ & $0(0.0)$ & $0(0.0)$ & 0.123 \\
\hline T1 (\%) & $10(20.4)$ & $3(9.4)$ & $2(6.2)$ & \\
\hline T2 (\%) & 14 (28.6) & $7(21.9)$ & 17 (53.1) & \\
\hline T3 (\%) & $7(14.3)$ & $6(18.8)$ & $4(12.5)$ & \\
\hline T4 (\%) & $17(34.7)$ & $16(50.0)$ & $9(28.1)$ & \\
\hline N0 (\%) & $12(24.5)$ & $6(18.8)$ & $7(22.6)$ & $0.029^{\star}$ \\
\hline N1a (\%) & $12(24.5)$ & $0(0.0)$ & $5(16.1)$ & \\
\hline N1b (\%) & $25(51.0)$ & $26(81.2)$ & 19 (61.3) & \\
\hline Age & 70.0 [27-91] & 73.0 [31-85] & 72.0 [41-85] & 0.912 \\
\hline Female (\%) & $29(56.9)$ & $22(68.8)$ & $23(65.7)$ & 0.502 \\
\hline Male (\%) & $22(43.1)$ & $10(31.2)$ & $12(34.3)$ & \\
\hline FTC $(\%)$ & $6(11.8)$ & $3(9.4)$ & $6(17.1)$ & 0.612 \\
\hline PTC (\%) & 45 (88.2) & $29(90.6)$ & $29(82.9)$ & \\
\hline Initial M (\%) & $23(45.1)$ & $8(25.0)$ & $14(40.0)$ & 0.179 \\
\hline FUD (y) & $3.90[0.4-21.0]$ & $5.20[0.7-16.4]$ & $6.80[1.8-15.0]$ & $0.014^{*}$ \\
\hline Tumor size ${ }^{\dagger}$ & $6.0[3-9]$ & 13.0 [10-26] & $17.0[10-48]$ & $<0.001^{*}$ \\
\hline $\mathrm{Tg}(\mathrm{ng} / \mathrm{mL})$ & $\begin{array}{c}24.6 \\
{[0.04-11,447]}\end{array}$ & $\begin{array}{c}58.6 \\
{[0.04-1700]}\end{array}$ & $\begin{array}{c}525.0 \\
{[0.2-180,000]}\end{array}$ & $0.002^{*}$ \\
\hline $\begin{array}{l}\text { Other M } \\
\qquad(\%)^{\ddagger}\end{array}$ & $14(27.5)$ & $11(34.4)$ & 22 (62.9) & $0.003^{*}$ \\
\hline Death (\%) & $1(2.0)$ & $5(15.6)$ & $4(11.4)$ & 0.071 \\
\hline
\end{tabular}

Classification of Tumors (T) and nodes $(\mathrm{N})$ was performed according to the seventh edition. ${ }^{\dagger}$ Largest diameter of pulmonary metastatic lesion.

${ }^{\ddagger}$ Other distant metastasis.

FTC indicates follicular thyroid carcinoma; FUD, follow-up duration after recognizing metastasis; M1, with pulmonary metastasis; PTC, papillary thyroid carcinoma; Tg, thyroglobulin level (recent in the follow-up group and baseline in lenvatinib group).

${ }^{\star} P<0.05$.
Kaplan-Meier method using the SPSS software (version 24; IBM Corp., Armonk, NY). The Kaplan-Meier estimator in the SPSS software was used to calculate OS and apply the log-rank test. Values with $P<0.05$ were considered statistically significant. The OS of the LM and LV groups was validated using the logrank test.

\section{Results}

As shown in Table 1, the median tumor size in the LV group (17 $\mathrm{mm}$ ) was significantly larger than that in the SM group (6 $\mathrm{mm}$ ) and in the LM group $(13 \mathrm{~mm})$, indicating disease progression. The serum thyroglobulin level was also significantly high, and other metastatic lesions were also more common in 22 patients $(62.9 \%)$ in the LV group. The follow-up period after pulmonary metastasis in the LV group was also the longest. Meanwhile, among the 32 patients in the LM group, 11 (34.4\%) had metastatic lesions with a maximum diameter of $\geq 15 \mathrm{~mm}$; their disease progression was apparent, and 5 of them died. Besides, 21 patients $(65.6 \%)$ had metastatic lesions measuring $10-14 \mathrm{~mm}$ in which the disease progression is still under observation. The 51 patients in the SM group, the metastatic lesions measured $<10$ $\mathrm{mm}$, and disease progression is slow or has been a stable disease for many years; however, 1 patient died of aspiration pneumonia (Fig. 1). In addition, 4 patients in the SM group had a serum thyroglobulin level of $<1.0 \mathrm{ng} / \mathrm{mL}$, and biologically, they had no evidence of disease ${ }^{[9]}$.

Considering disease progression, 35 patients were treated with lenvatinib, while 4 died of cancer-specific disease. Meanwhile, lenvatinib treatment could not be continued in 2 patients because of having adverse events, hemorrhagic gastric ulcer, and persistent severe skin disorder; in one patient because of depression related to lenvatinib treatment, and in another patient because of suspected anaplastic transformation due to progressive disease

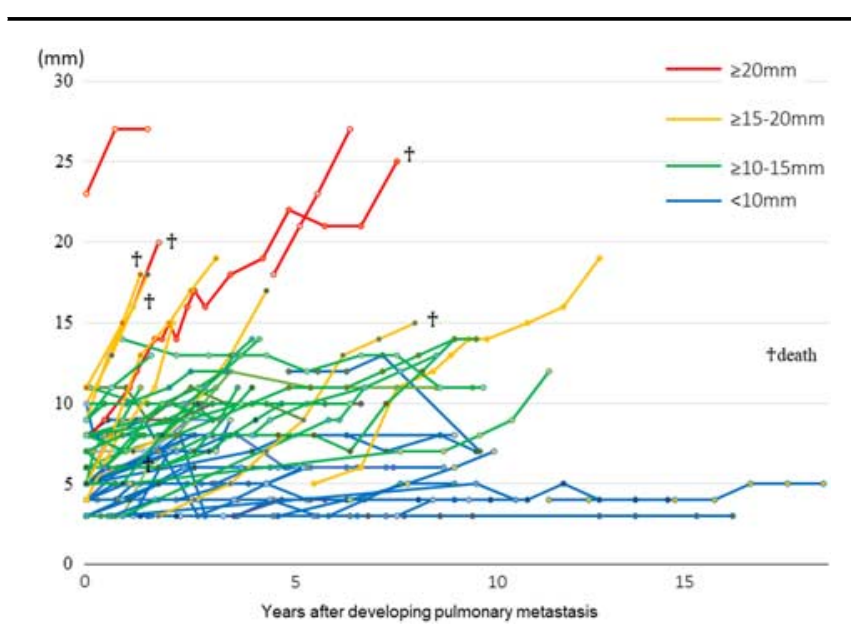

Figure 1. Tumor size changes after the pulmonary metastasis appearance in 83 follow-up cases (small metastasis group and large metastasis group). 4 : death cases. Red: patients with $\geq 20 \mathrm{~mm}$ metastatic lesion in which 2 of 4 patients died. Yellow: patients with 15-19 mm metastatic lesion in which 3 of 7 patients died. Green: 21 patients with 10-14 mm metastatic lesion in which none of them died. Blue: 51 patients with $\leq 9 \mathrm{~mm}$ metastatic lesion in which 1 patient died from non-cancer-specific disease but the disease progression has not been observed. 

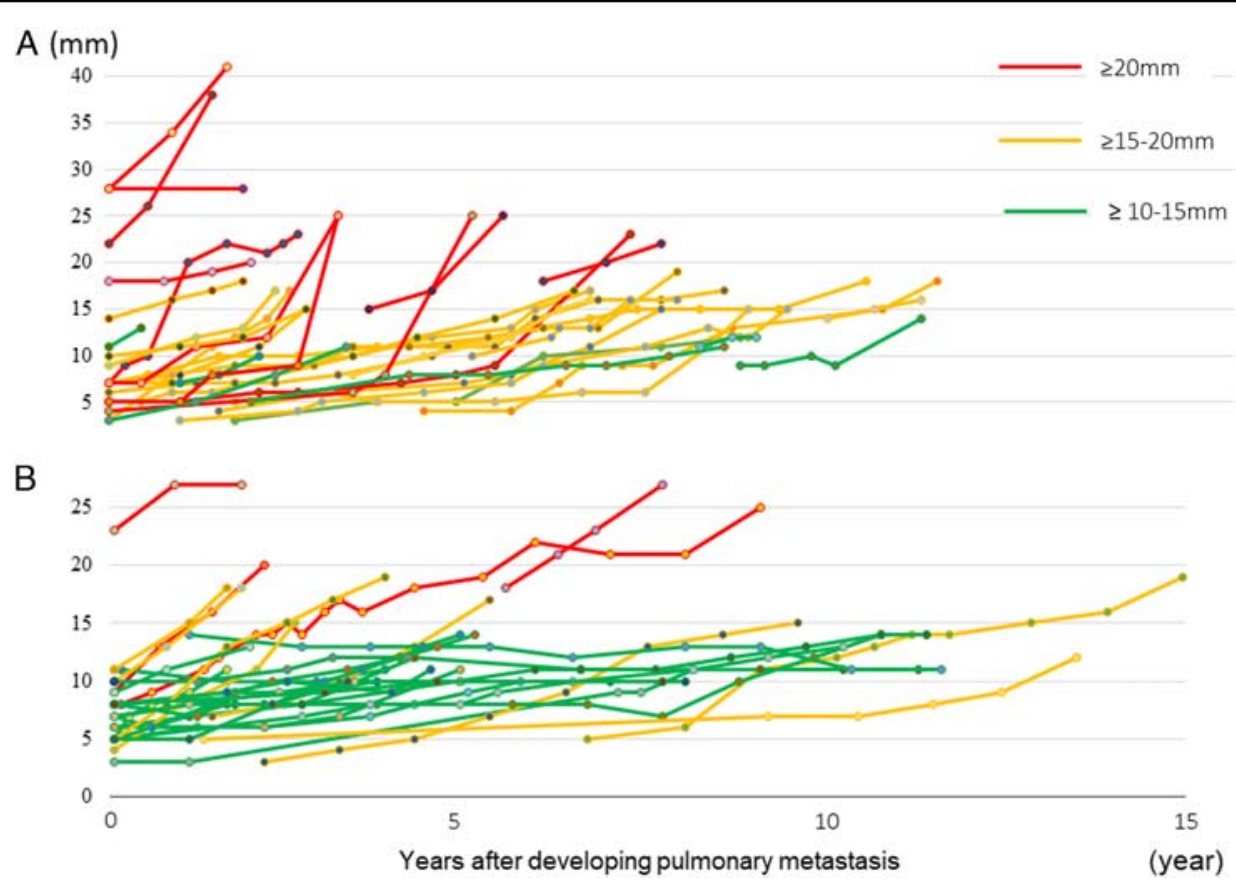

Figure 2. Tumor size changes before treatment in the lenvatinib treatment and large metastasis groups. The large metastasis group included cases in which lenvatinib treatment was not indicated because of no disease progression and in those who refused treatment. Red: 11 patients with $\geq 20 \mathrm{~mm}$ metastatic lesion. Yellow: 18 patients with 15-19 mm metastatic lesion. Green: 6 patients with 10-14 mm metastatic lesion. The larger the tumor diameter, the faster it grows.

(PD). Subsequently, the treatment evaluations according to RECIST guidelines ${ }^{[7]}$ were PD in 2, stable disease (SD) in 11, and partial response (PR) in 22 patients. Figure 2 shows the progression of the metastatic lesion size in the LM and LV groups before treatment. The disease clearly progressed rapidly in all patients in the LV group. Lenvatinib treatment was started when the maximum diameter of metastases was $\geq 20 \mathrm{~mm}$ in 11 $(31.4 \%)$ patients, $15-19 \mathrm{~mm}$ in $18(51.4 \%)$ patients, and $10-14$ $\mathrm{mm}$ in $6(17.1 \%)$ patients. The posttreatment progress is shown in Figure 3. Of 29 patients with $\geq 15 \mathrm{~mm}$ metastatic lesions, 4 died because of failed treatment continuity due to the presence of adverse event and other reasons; meanwhile, all 6 patients with 10-14 mm metastatic lesions survived. The metastatic lesions in $16(45.7 \%)$ patients have shrunk to $<10 \mathrm{~mm}$. Figure 4 illustrates a waterfall graph of the therapeutic effect. The OS curves of the LM and LV groups are shown in Figure 5. Although no significant difference $(P=0.334)$ was observed, the 10 -year survival rate was $84.3 \%$ in the LV group and $70.6 \%$ in the LM group, and the curve was higher even if the disease progressed clearly. The median treatment period was 23.0 (4.2-61.2) months, the initial dose was 24 (10-24) mg, and the current dose was 10.0 (8-14) $\mathrm{mg}$. The reduction ratio shown in the graph is not the best response but the current evaluation. Among the 31 patients with ongoing treatment, $9(29.0 \%)$ had SD, and $22(71.0 \%)$ patients had PR.

\section{Discussion}

Tumor burden can be accurately determined by measuring the tumor diameter in pulmonary metastases. In each patient, considering that the disease progression can be evaluated precisely by the unit $\mathrm{mm}$, the prognosis of DTC with pulmonary metastasis can be appropriately assessed. Of the 32 patients in the LM group, 11 patients with $\geq 15 \mathrm{~mm}$ metastatic lesions experienced refractory disease progression, and 5 of them died. These results are consistent with the results obtained before the TKI treatment introduction $^{[10]}$. Therefore, if the metastatic lesion is $\geq 15 \mathrm{~mm}$, the disease is life threatening; without lenvatinib treatment, the affected patients will die from disease progression. Although most pulmonary metastases from DTC have a favorable prognosis, they are definitely fatal as the disease progresses ${ }^{[9]}$. In 21 patients with $10-14 \mathrm{~mm}$ metastatic lesions, active surveillance will be necessary in the future. Some patients seem to have PD, while some exhibited an SD. The remaining 51 (66.3\%) patients in the SM group with $<10 \mathrm{~mm}$ metastatic lesions had a slow disease progression or SD for many years according to the current treatment strategy. In other words, RAI treatment and TSH suppression may be successful. Therefore, even if there is pulmonary metastasis, if it is $<10 \mathrm{~mm}$, it does not affect the life prognosis.

Meanwhile, 35 patients who were RAI refractory, had disease progression and were treated with lenvatinib. As shown in Figures 3 and 5, evaluation on disease progression before and after lenvatinib treatment revealed that the tumor reduced with lenvatinib regardless of the initial size of pulmonary metastases and that SD and PR could be maintained for a long time. Except for four deaths, $31 / 35(88.6 \%)$ patients showed that lenvatinib suppressed disease progression and prolonged patient survival. Unfortunately, several disease complications, such as bone metastases, brain metastases, local recurrences, mediastinal lymph node recurrences, and pleural dissemination, do not improve treatment results easily ${ }^{[9,11]}$. Currently, the 10 - and 20 -year survival rates are $95.1 \%$ and $86.4 \%$, respectively, in our hospital for DTC patients with distant metastasis. These 


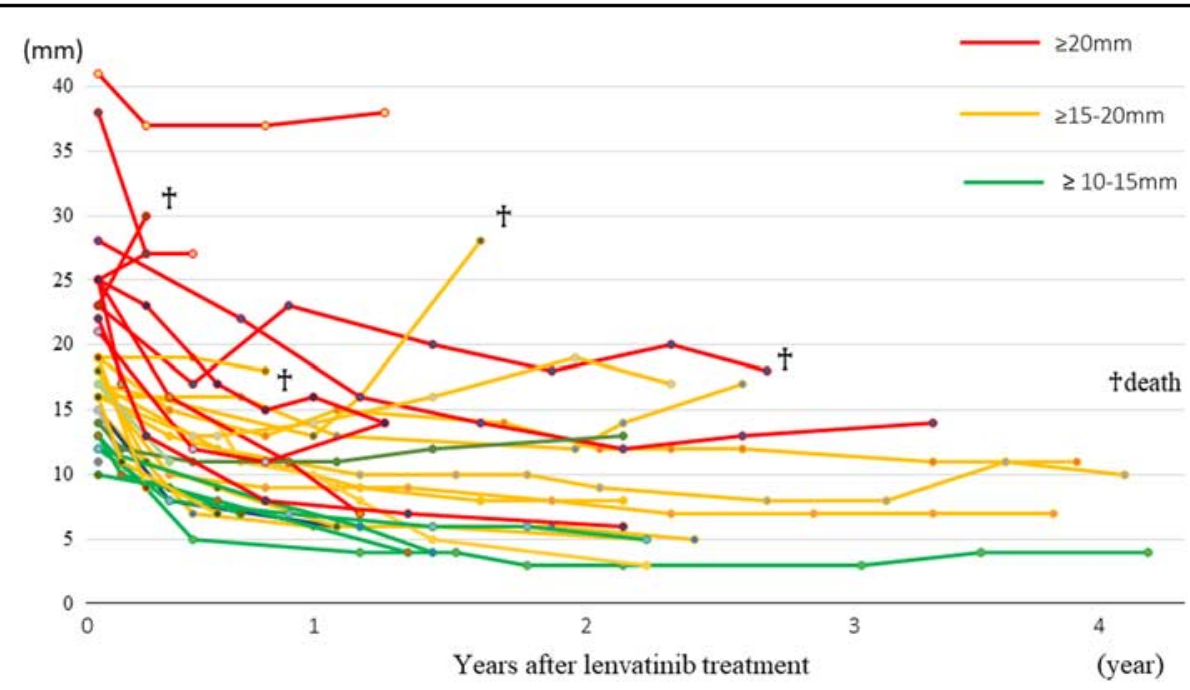

Figure 3. Progress of metastatic lesions by lenvatinib treatment. was $\geq 20 \mathrm{~mm} ; 2$ patients died. Yellow: 18 patients who started treatment when the metastatic lesion size was 15-19; 2 patients died. Green: 6 patients who started treatment when the metastatic lesion size was $10-14 \mathrm{~mm}$; none of them died. Disease progression is suppressed in all patients by continual lenvatinib treatment.

improved outcomes for DTC patients with distant metastases are driven by favorable outcomes for the most common pulmonary metastases.

In the real world, lenvatinib treatment results are reportedly worse ${ }^{[12,13]}$ than those in the SELECT trial ${ }^{[14]}$. Nonetheless, the results in this study are favorable because we excluded patients with bone metastases or local disease for the target lesion. However, the following limitations should be considered. First, all pulmonary metastases could not be evaluated with a maximum diameter by the unit $\mathrm{mm}$. Some patients have diffuse or numerous nodules in pulmonary metastasis ${ }^{[15]}$. In these patients, disease progression should be evaluated by the increase of the tumor volume on the image. Although some patients with diffuse pulmonary metastases are included in this study, delayed evaluation of disease progression and missed the timing of lenvatinib treatment must be avoided. Second, regarding pleural effusion

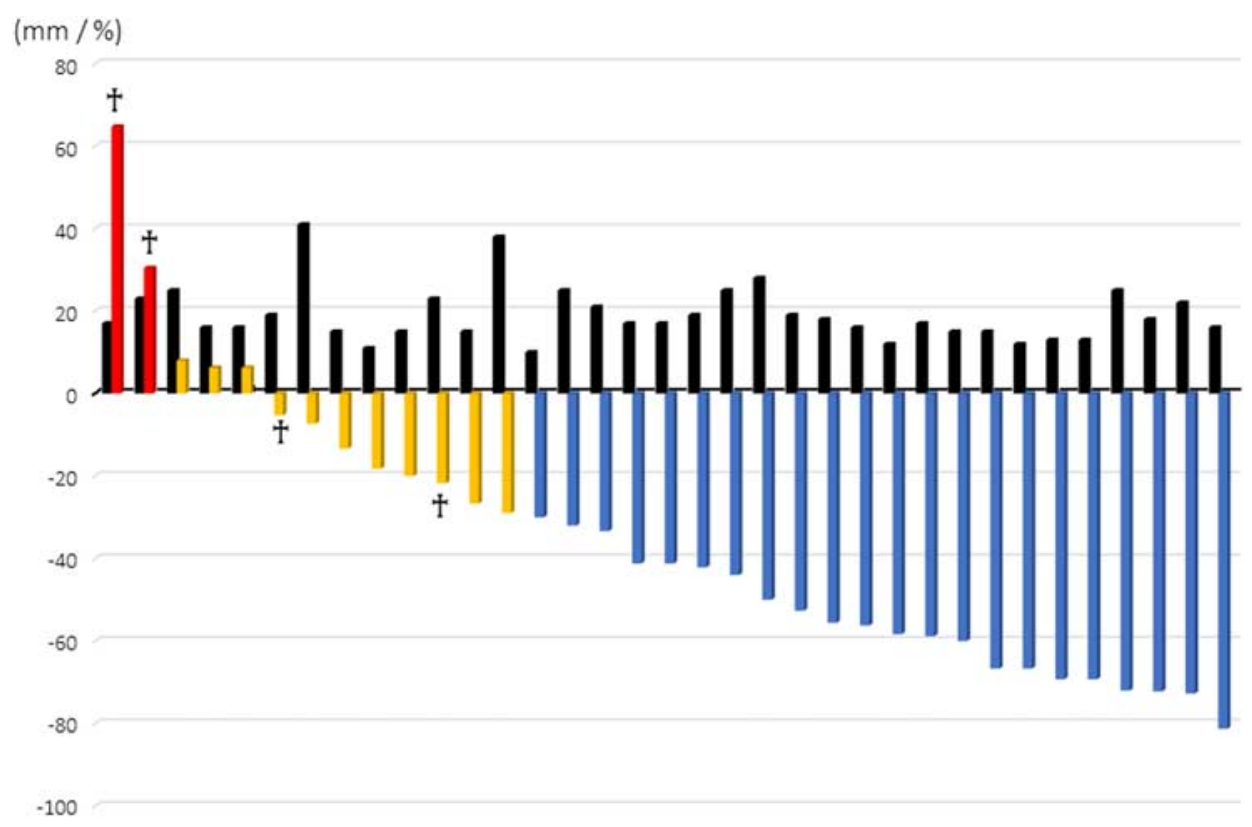

$(\%)$

Figure 4. Baseline tumor size before lenvatinib treatment and current tumor reduction rate. Black: baseline tumor diameter (mm). The graph contains the reduction rate of progressive disease (PD; red), stable disease (SD; yellow), and partial response (PR; blue). The reduction ratio is not the best response but the current size. Units are \% divided by baseline tumor size. Furthermore, 2 patients with PD, and 2 patients with SD died because they could not continue their oral administration. Of the 31 patients who continued oral administration, 9 (29.0\%) had SD, and 22 (71.0\%) had PR. 


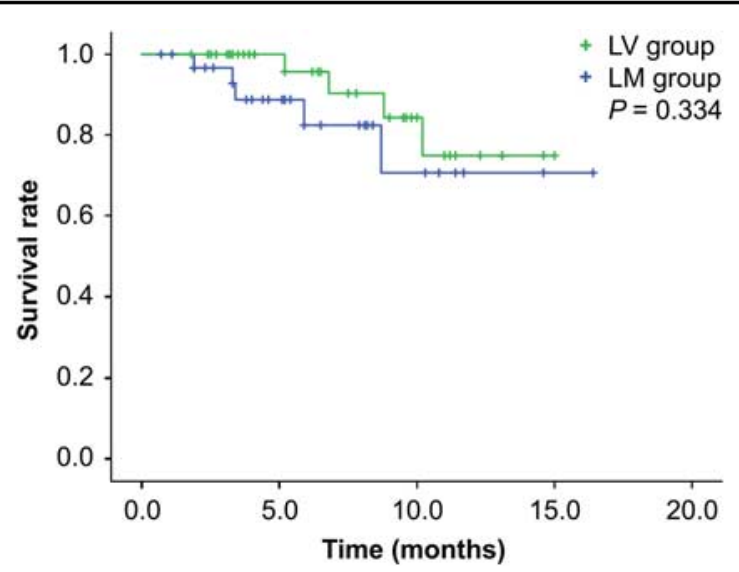

Figure 5. Comparison of OS curves after the appearance of lung metastases between LM and LV groups. No significant difference was noted $(P=0.334)$, but the curve for the LV group is located above. It is suggested that lenvatinib prolongs survival even in the LV group, in which the disease progression was apparently progressing. LM indicates large metastasis, LV; lenvatinib treatment.

with coexisting pleural dissemination, mediastinal, hilar lymph node metastases, and pleural dissemination were excluded because it may be symptomatic regardless of the tumor diameter; once pleural effusion is recognized, the tumor diameter cannot be measured. Considering that these patients have a poor prognosis, establishing another standard for evaluating disease progression is necessary.

Our study has some limitations. First, our study is nonrandomized; however, randomized studies of patients with rapidly progressive, iodine refractory thyroid cancer are difficult to perform. Second, it is difficult to establish whether lenvatinib suppressed disease progression and prolonged survival. Our data may suggest it, but there is no clear proof.

\section{Conclusion}

Overall, in the retrospective evaluation of the prognosis of 118 DTC patients with pulmonary metastases indicating disease progression, 35 were treated with lenvatinib, and 29 of them had metastatic lesions measuring $\geq 15 \mathrm{~mm}$, which was life threatening. Results showed that lenvatinib reduced the disease progression and contributed to survival extension in 31 of the 35 patients.

\section{Ethics approval and consent to participate}

The Chemotherapy Committee of Kanagawa Cancer Center approved the lenvatinib treatment regimens administered to patients with DTC in this study. The Cancer Board of the hospital also approved these TKI treatments, including surgery, for patients with DTC who were included. The study was approved by the Institutional Review Board of Kanagawa Cancer Center (IRB approval number: 27-61).

\section{Sources of funding}

No funding was received.

\section{Author contribution}

H.I.: prepared the manuscript. H.I., S.T., and D.M.: designed the study. D.M. and A.M.: analyzed the data. All authors read and approved the final manuscript.

\section{Conflict of interest disclosure}

H.I. is an endocrine surgeon working at the Kanagawa Cancer Center and has extensive experience with several surgeries for advanced thyroid cancer as well as anaplastic treatment of thyroid cancer. The remaining authors declare that they have no financial conflict of interest with regard to the content of this report.

\section{Research registration unique identifying number (UIN)}

Not applicable.

\section{Guarantor}

Not applicable.

\section{Acknowledgment}

The authors thank Dr Hiroyuki Hayashi (Department of Pathology, Yokohama City Hospital) for pathologic diagnosis.

\section{References}

[1] Davies L, Welch HG. Current thyroid cancer trends in the United States. JAMA Otolaryngol Head Neck Surg 2014;140:317-22.

[2] Haugen BR. 2015 American Thyroid Association management guidelines for adult patients with thyroid nodules and differentiated thyroid cancer: What is new and what has changed? Cancer 2017;123:372-81.

[3] Goffredo P, Sosa JA, Roman SA. Differentiated thyroid cancer presenting with distant metastases: a population analysis over two decades. World J Surg 2013;37:1599-605.

[4] Kelly A, Barres B, Kwiatkowski F, et al. Age, thyroglobulin levels and ATA risk stratification predict 10-year survival rate of differentiated thyroid cancer patients. PLoS One 2019;14:e0221298.

[5] Lang BH, Wong KP, Cheung CY, et al. Evaluating the prognostic factors associated with cancer-specific survival of differentiated thyroid carcinoma presenting with distant metastasis. Ann Surg Oncol 2013;20: 1329-35.

[6] Sampson E, Brierley JD, Le LW, et al. Clinical management and outcome of papillary and follicular (differentiated) thyroid cancer presenting with distant metastasis at diagnosis. Cancer 2007;110:1451-6.

[7] Eisenhauer EA, Therasse P, Bogaerts J, et al. New response evaluation criteria in solid tumours: revised RECIST guideline (version 1.1). Eur J Cancer 2009;45:228-47.

[8] Kanda Y. Statistical analysis using freely-available "EZR (Easy R)" software. Rinsho Ketsueki 2015;56:2258-66.

[9] Tuttle RM, Tala H, Shah J, et al. Estimating risk of recurrence in differentiated thyroid cancer after total thyroidectomy and radioactive iodine remnant ablation: using response to therapy variables to modify the initial risk estimates predicted by the new American Thyroid Association staging system. Thyroid 2010;20:1341-9.

[10] Lee J, Soh EY. Differentiated thyroid carcinoma presenting with distant metastasis at initial diagnosis clinical outcomes and prognostic factors. Ann Surg 2010;251:114-9.

[11] Iwasaki H, Yamazaki H, Takasaki H, et al. Treatment outcomes of differentiated thyroid cancer with distant metastasis improve by tyrosine kinase inhibitors. Oncol Lett 2019;17:5292-300.

[12] Locati LD, Piovesan A, Durante C, et al. Real-world efficacy and safety of lenvatinib: data from a compassionate use in the treatment of radioactive 
iodine-refractory differentiated thyroid cancer patients in Italy. Eur J Cancer 2019;118:35-40.

[13] Aydemirli MD, Kapiteijn E, Ferrier KRM, et al. Effectiveness and toxicity of lenvatinib in refractory thyroid cancer: Dutch real-life data. Eur J Endocrinol 2020;182:131-8.
[14] Schlumberger M, Tahara M, Wirth LJ, et al. Lenvatinib versus placebo in radioiodine-refractory thyroid cancer. N Engl J Med 2015;372:621-30.

[15] Xiang MM, Qin JY, Shen YC, et al. Lung metastasis of thyroid cancer appearing diffuse and disseminated on imaging. Chin Med J (Engl) 2018;131:2132. 